\title{
Antireflection High-Index Metasurfaces Combining Mie and Fabry-Pérot Resonances
}

Cordaro, Andrea; Van De Groep, Jorik; Raza, Søren; Pecora, Emanuele Francesco; Priolo, Francesco; Brongersma, Mark L.

\section{Published in:}

ACS Photonics

Link to article, DOI:

10.1021/acsphotonics.8b01406

Publication date:

2019

Document Version

Peer reviewed version

Link back to DTU Orbit

Citation (APA):

Cordaro, A., Van De Groep, J., Raza, S., Pecora, E. F., Priolo, F., \& Brongersma, M. L. (2019). Antireflection High-Index Metasurfaces Combining Mie and Fabry-Pérot Resonances. ACS Photonics, 6(2), 453-459. https://doi.org/10.1021/acsphotonics.8b01406

\section{General rights}

Copyright and moral rights for the publications made accessible in the public portal are retained by the authors and/or other copyright owners and it is a condition of accessing publications that users recognise and abide by the legal requirements associated with these rights.

- Users may download and print one copy of any publication from the public portal for the purpose of private study or research.

- You may not further distribute the material or use it for any profit-making activity or commercial gain

- You may freely distribute the URL identifying the publication in the public portal 


\title{
Anti-reflection high-index metasurfaces combining
}

\section{Mie and Fabry-Pérot resonances}

\author{
Andrea Cordaro ${ }^{1,2,3 \dagger}$, Jorik van de Groep ${ }^{1}$, Søren Raza ${ }^{1,4}$, Emanuele F. Pecora ${ }^{1}$, Francesco \\ Priolo $^{2,3}$ and Mark L. Brongersma ${ }^{1 *}$
}

${ }^{1}$ Geballe Laboratory for Advanced Materials, Stanford University, Stanford, California 94305, United States

${ }^{2}$ Dipartimento di Fisica e Astronomia, Università di Catania, via S. Sofia, 64, 95123 Catania, Italy

${ }^{3}$ Scuola Superiore di Catania, Università di Catania, via Valdisavoia, 9, 95123 Catania, Italy

${ }^{4}$ Department of Physics, Technical University of Denmark, DK-2800 Kongens Lyngby, Denmark

*brongersma@stanford.edu

KEYWORDS. Metasurface, Anti-Reflection Coating (ARC), Mie resonators, Effective Medium, Fabry-Pérot resonances

ABSTRACT. Minimizing reflection losses is required for the efficient operation of a wide range of optical components. Anti-reflection coatings supporting Fabry-Pérot resonances are commonly used to solve this problem and can be applied on an industrial scale. Recent work has shown that 
reflections can also be reduced by placing an array of high-index nanostructures on a surface. In such coatings, anti-reflection is achieved by tailoring the scattering by optical Mie resonances. Here, we design and fabricate Si metasurfaces that combine both Fabry-Pérot and Mie resonances in a single metasurface to realize a multi-resonant broadband anti-reflection response. We optically characterize the metasurfaces demonstrating 4.1\% AM1.5-averaged reflectance across the visible spectrum (425-900 nm). Our metasurface design strategy is generally applicable to different materials and frequency ranges, making our approach relevant for a broad variety of applications.

\section{Introduction}

The quest for more efficient photonic devices relies on our ability to manage the flow of light at the nanoscale. In this work, we analyze whether Mie resonant nanostructures can help reduce unwanted reflections at materials interfaces, arguably the most ubiquitous problem in the design of optoelectronic devices. For photovoltaic applications, the design of anti-reflection (AR) coatings is particularly challenging as it needs to be achieved across a very broad spectral range.

The minimization of reflection of a flat substrate was first demonstrated by placing a thin layer of transparent dielectric - referred to as Rayleigh's film ${ }^{1}$ - at the interface of the substrate to suppress reflection by destructive interference ${ }^{2}$. However, a major disadvantage of such singlelayer interference coatings lies in the strong dependence on both the angle of incidence and the wavelength of the incident light. Further evolutions of the interference AR concept include multiple or graded-index coatings ${ }^{3-7}$, achieving broadband and omnidirectional AR at the expense of ease of fabrication and costs.

Novel approaches exploit high-index resonant scatterers ${ }^{8}$ and optical metasurfaces ${ }^{9,10}$ to achieve broadband anti-reflection. These are arrays of low-aspect-ratio nanostructures that can 
conveniently be patterned by a variety of approaches. When properly sized, they can effectively capture and redirect light through the excitation of optical Mie resonances ${ }^{11-15}$. This enables strong forward scattering ${ }^{16,17}$ of incident light into a substrate to reduce reflectance ${ }^{18,19}$. However, the spectral efficiency of these nanopatterns is limited by their resonance bandwidth and a multiresonant behavior is needed in order to cover the majority of the solar spectrum ${ }^{8,20}$.

Here, we demonstrate how silicon (Si) metasurfaces can combine the beneficial effects of FabryPérot (FP) resonances and Mie resonances in a single layer. To this end, we carefully arrange nanowires or pillars into sub-wavelength arrays to achieve broadband and spectrally engineered anti-reflection. Using a simple theoretical model based on coupled mode theory (CMT) ${ }^{21-25}$, we develop an intuitive insight into the multi-modal interaction between the FP and Mie resonances within the nanopatterned layer. Our experimental results demonstrate broadband anti-reflection in the spectral range from $425-900 \mathrm{~nm}$ for optimized designs. The multi-resonant metasurface concept can be generalized to all high-index materials and other wavelength ranges, making it suitable for a broad range of applications including solar cells, sensors, and other optical components.

\section{Theory}

In this section, we introduce the concept of a multi-resonant metasurface in which FP and Mie resonances can be made to work together to reduce reflection. The initial starting point is to leverage the work on conventional thin interference AR coatings to achieve a low reflectance across a broad range of wavelengths in the red side of the solar spectrum. Then, we will use the knowledge of Mie resonators to carve a second reflectance minimum at shorter wavelengths in order to achieve an overall more broadband antireflection in a single effective layer. 
Whereas traditional AR coatings have been continuous films of a single material (e.g. $\mathrm{Si}_{3} \mathrm{~N}_{4}$ ), more flexibility to control the refractive index has come from the use of deep-subwavelength nanostructures forming a metamaterial with an effective index. To illustrate this concept, we first consider a dense array of Si nanobeams placed on a Si substrate. When the spacing of the nanobeams is made sufficiently small compared to the illumination wavelength, the array functions as an effective medium supporting a FP resonance. The location of this resonance depends on the layer's effective index $n_{\text {eff }}$ that is controlled by the refractive indices of the constituents ( $n_{\text {air }}$ and $\left.n_{\mathrm{si}}\right)$ as well as by their filling fractions ${ }^{26}$. To first order, the magnitude of $n_{\text {eff }}$ is described by effective medium theory. For anisotropic nanobeam arrays, the effective index depends on the polarization of incoming light as ${ }^{27}$ :

$$
n_{\mathrm{eff}}^{\perp}=\left(\frac{1-F}{n_{\mathrm{air}}^{2}}+\frac{F}{n_{\mathrm{Si}}^{2}}\right)^{-1 / 2} n_{\mathrm{eff}}^{\|}=\left[(1-F) n_{\mathrm{air}}^{2}+F n_{\mathrm{Si}}^{2}\right]^{1 / 2}
$$

where $n_{\mathrm{eff}}^{\perp}$ is used when the electric field $\mathbf{E}$ is polarized perpendicularly to the wire axes (TE polarization) and $n_{\text {eff }}^{\|}$is used when $\mathbf{E}$ is polarized along the wire axes (TM), and $F$ is the filling fraction of Si. For most practical purposes a polarization-independent geometry based on, e.g., nanopillars is more relevant. In the latter case, the effective index is given by the root mean square of $n_{\text {eff }}^{\perp}$ and $n_{\text {eff }}^{\| 2}$. To highlight the key physics, we first consider a constant refractive index $n_{\mathrm{Si}}=$ $4+0.1 i$ to avoid complications from the material dispersion in real Si. Figure 1a shows the analytically calculated reflectance of a slab of thickness $h=120 \mathrm{~nm}$ with index given by Eq. (1) (with $n_{\text {air }}=1$ ) as a function of the filling fraction (directly related to the periodicity). Reducing the period from $300 \mathrm{~nm}$ to $120 \mathrm{~nm}$ for fixed wire width $(80 \mathrm{~nm})$ shifts the minimum to 
(a) Effective Medium ARC
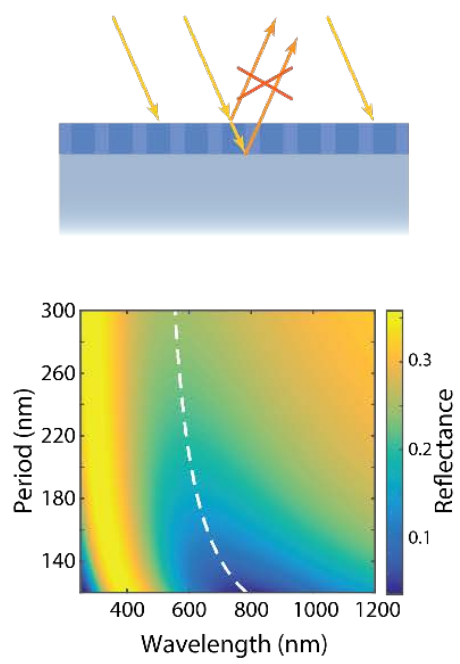

(b)
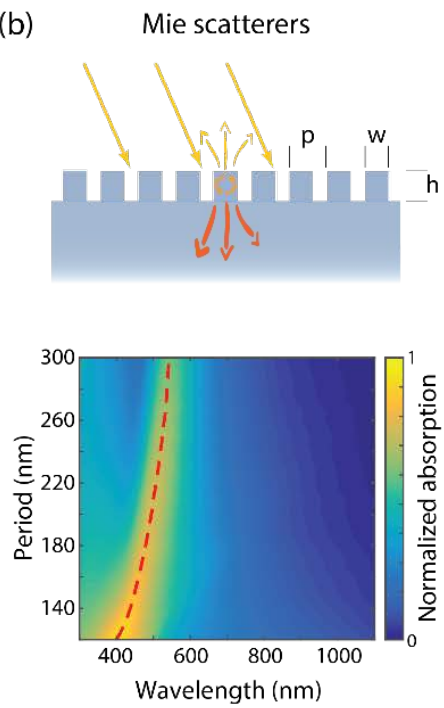

(c)

Combined Effects

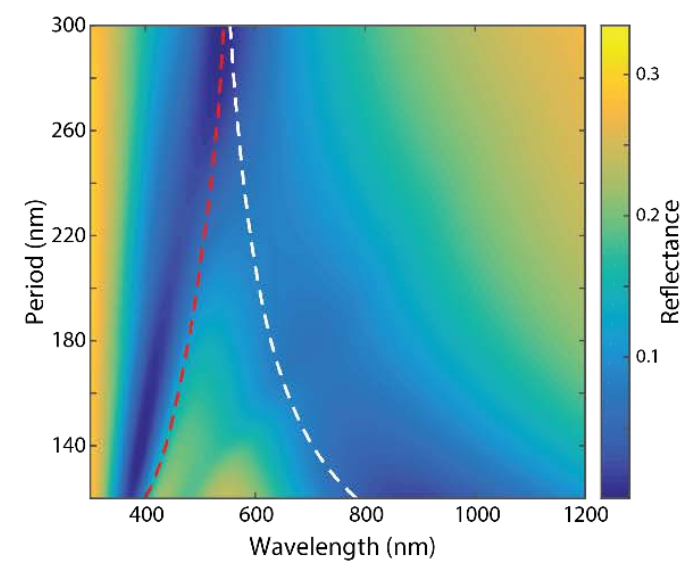

Figure 1. Antireflection behavior of Mie-resonant antireflection coatings supporting Fabry-Pérot and Mie-resonances. (a) Analytically calculated reflectance of a slab of index $n_{\text {eff }}$ on a substrate with index $n_{\mathrm{Si}}=4+0.1 i$ as a function of $\mathrm{p}$ (and thus $n_{\mathrm{eff}}$ ) and $\lambda$. (b) The importance of a Mie resonance is seen in the simulated spectral absorption in Si nanobeam arrays placed on a Si substrate. A schematic of the structure illustrates the excitation of a Mie resonator. The beam width in chosen as $80 \mathrm{~nm}$ and the height as $120 \mathrm{~nm}$ for all of the panels in this figure. (c) Simulated reflectance of an array of Si nanowires on a substrate as a function of $\mathrm{p}$ and $\lambda$.

longer wavelengths. At the same time, the minimum becomes lower as $n_{\text {eff }}$ increases and gets closer to the ideal AR condition $n_{\mathrm{eff}}=\sqrt{n_{\mathrm{air}} n_{\mathrm{Si}}}$. The interference coating thus forms a single broad dip in reflection as a result of a broad Fabry-Pérot resonance.

Recent work has demonstrated that high-index nanostructures with sizes comparable to the wavelength of light in the medium support optical Mie-type resonances ${ }^{11-15}$ whose spectral location can be tuned with both the nanobeam height and width ${ }^{29}$. For 80 -nm-wide and 120 -nmhigh beams, the lowest-order Mie resonance (Magnetic Dipole) appears near the peak of the solar 
spectrum at $500 \mathrm{~nm}^{17}$ (Fig. 1b). As a result, light at this wavelength is efficiently scattered forward into the substrate, lowering reflection.

According to the above discussion, it is clear that a dense array of nanobeams can support two pathways for an incident light wave: a FP resonant pathway governed by the effective-index layer just described (Fig. 1a) and a Mie-resonant pathway (Fig. 1b). In order to investigate the interplay of the two pathways, Fig. 1c shows the simulated (Lumerical FDTD ${ }^{30}$ ) reflectance spectra of an array of nanobeams under normal incidence (TE polarized) as a function of the periodicity $p$. Only one reflection minimum is observed at larger periodicities, while another dip appears as the array periodicity is reduced. This provides for a broader and more efficient anti-reflection behavior.

To identify the origin of the two minima, two dashed lines are added to Fig. 1c indicating the resonance wavelength of the isolated mechanisms (Figs. 1a, b). The red dashed line marks the peak of the power absorbed within the wires (Fig. 1b), which is a good indication of the system's Mie resonance wavelength as a function of the array periodicity. For small periods, the magnetic dipole modes in neighboring wires couple, giving rise to a blue-shift. Hence the red dashed line indicates the spectral location where the Mie resonant pathway dominates. On the other hand, the dashed white line (Fig. 1a) indicates the reflectance minimum as a function of filling fraction and helps to identify the FP pathway in Fig. 1c. Based on the broader spectral properties of the FP resonance, we will refer to the FP related feature as the Fabry-Pérot background.

It is important to stress that the position of the minima in Fig. 1c does not necessarily coincide with the minima that the two pathways would generate individually. In fact, the interference of the broad FP background and the Mie resonance can give rise to a characteristic asymmetric Fano line-shape ${ }^{31}$. For such a resonance, the distance between the minimum of the spectrum and 
(a)

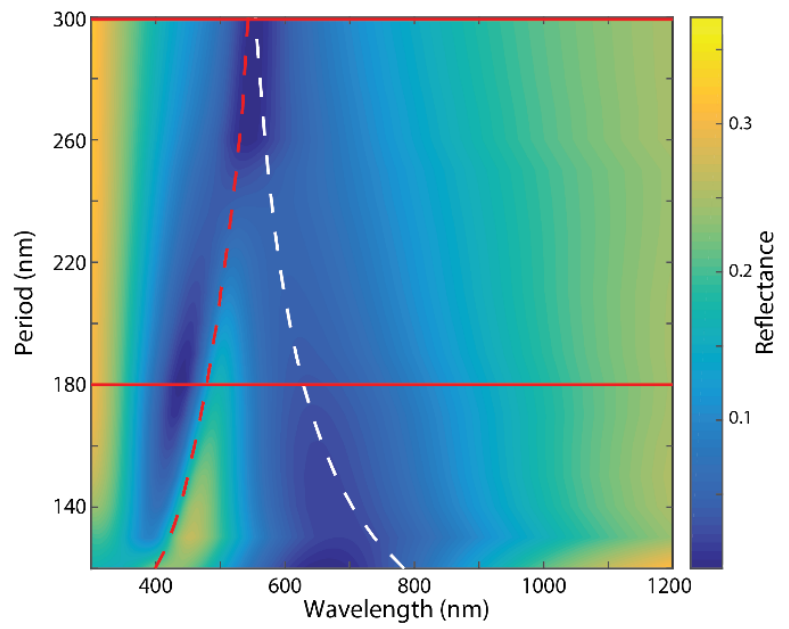

(b)

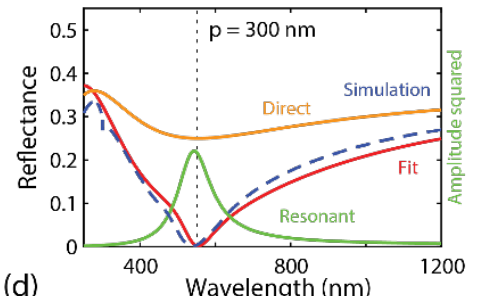

(d)

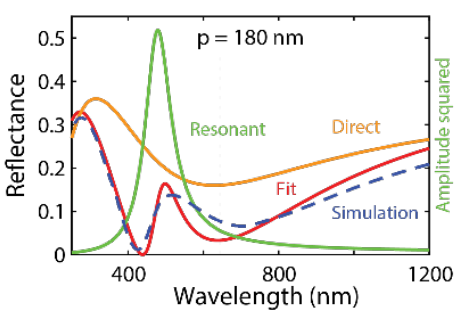

(c)

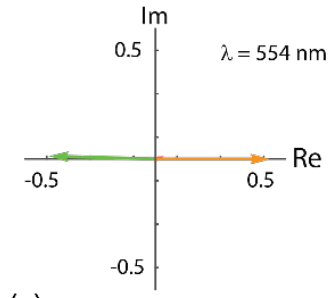

(e)

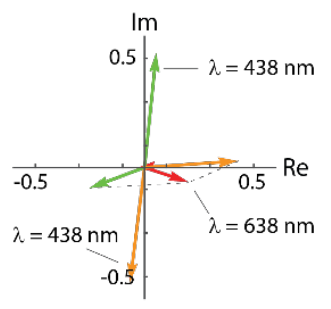

Figure 2. Coupled mode theory for Mie-resonant antireflection coatings. (a) CMT-modeled reflectance map obtained by fitting to the spectral reflection data obtained by FDTD simulations as shown in Fig. 1c. (b,d) Comparison between simulated (dashed blue) and CMT-fitted spectra (red) for an array of nanowires ( $\mathrm{w}=80 \mathrm{~nm}, \mathrm{~h}=120 \mathrm{~nm}$ ) for $\mathrm{p}=300 \mathrm{~nm}$ (b) and p = $180 \mathrm{~nm}$ (d). Square modulus of the direct (yellow) and resonant (green) pathways composing the fits are also shown. Note that the label "Reflectance" does not apply to the resonant pathway. (c,e) Phasor diagram representation of the two pathways at the wavelengths of the minima (dashed lines) in (b,d).

the resonant frequency depends on the so-called Fano shape parameter, which describes the degree of asymmetry ${ }^{32,33}$. Moreover, the second minimum is slightly shifted compared to that of the FP background alone due to the presence of the Mie resonance. To achieve the best antireflection, it is clear that the relative amplitude and phase of the light scattered fields produced by each mechanism will need to be optimized. We use coupled mode theory to quantitatively explore the interaction of the two resonant mechanisms. CMT is very powerful in describing the behavior of coupled resonances and enables the possibility of calculating the reflectance of an optical system by modeling its interaction with incident light through different resonant processes ${ }^{21-25}$. The reflectance of the coupled system is given by (see Supplementary Information): 


$$
R=\left|r_{\mathrm{eff}}+\frac{A e^{i \phi}}{i\left(\omega-\omega_{0}\right)+g}\right|^{2}
$$

Here, $r_{\text {eff }}$ is the Fresnel reflection coefficient for a dielectric slab of index $n_{\text {eff }}$ on a substrate. The parameters $\omega_{0}, A, \phi$ and $g$ determine the resonance frequency, amplitude, phase offset and width of the Lorentzian function in the second term. The first term represents the broader FP background while the second term corresponds to the Mie-resonant pathway with a Lorentzian lineshape. To capture the antireflection behavior over a broader wavelength range or with different particle sizes, additional Mie resonances may be added.

To demonstrate that the doubly-resonant nature observed in Fig. 1 is well-described by CMT, we explore whether the full-field simulated spectra can be captured by Eq. (2). A quick inspection of Eq. (2) reveals that the dependent parameters are $n_{\text {eff, }} \omega_{0}, A, \phi$ and $g$. Given the large number of parameters, it is of value to obtain physically reasonable values for as many of them as possible. As mentioned, $n_{\text {eff }}$ is a function of the Si filling fraction and a reasonable estimate of $\omega_{0}$ can be obtained from the frequency of maximum absorption within the wires (Fig. 1b). Based on these assumptions, the spectra composing Fig. 1c are then fitted to generate Fig. 2a. Despite its simplicity and approximations (see Supplementary Information), the agreement between the CMT model and full-field simulations across many periods and wavelengths suggests that it captures the key physics. As such, it is a useful tool to optimize the broadband antireflection of these types of coatings.

Due to the complex-valued nature of the two terms in Eq. (2), anti-reflection behavior at multiple spectral locations is not obtained by simply adding two pathways that would generate a dip in reflectance when isolated (in accordance with the observations in Fig. 1). To fully exploit the doubly resonant nature of our structure, the amplitude of the two pathways must match with a $\pi$ 
phase difference. To evaluate whether these conditions are met, two spectra in the single-dip and double-dip regime are isolated $(\mathrm{p}=300 \mathrm{~nm}$ and $\mathrm{p}=180 \mathrm{~nm}$, respectively) and plotted along with the amplitude of the corresponding FP (yellow) and Mie resonant (green) pathways (Figs. 2b, d). The spectra obtained from the full-field simulations are also shown (blue dashed), demonstrating good correspondence with the CMT fit (red). For p = $300 \mathrm{~nm}$ (single-dip regime) the FP and Mie resonant pathways are almost equal in amplitude on resonance ( $\lambda=554 \mathrm{~nm}$, Fig. $2 \mathrm{~b})$. To study the relative phase at resonance, a phasor diagram is used to simultaneously plot the amplitude and phase for the two pathways in the complex plane (Fig. 2c). The resulting reflectance (red) can be obtained by vectorial addition of the direct (yellow) and resonant (green) components. Fig. 2c clearly demonstrates that the two pathways are close to equal in amplitude and $\pi$ out of phase simultaneously, giving rise to a low reflectance. For the period of $180 \mathrm{~nm}$, a double-dip occurs and the interference between the two pathways gives rise to a characteristic asymmetric Fano lineshape superimposed on the broad FP background. The amplitude is matched twice (Fig. 2d) but the AR condition is perfectly satisfied only for the first dip $(\lambda=438 \mathrm{~nm})$. The second dip is slightly red-shifted with respect to the second amplitude-matching wavelength, and the phase difference between the pathways is slightly less than $\pi$ (Fig. 2e). As a result, the reflection is not completely cancelled.

Finally, both resonant mechanisms - and therefore the entire reflectance spectrum - can be designed at will through careful engineering of the nanostructure dimensions (see Supplementary Info). For example, increasing the height of the wires results in a thicker effective layer, shifting the dip in the Fabry-Pérot background to longer wavelengths. On the other hand, changing the dimension of the nanobeams also results in a change in the Mie resonant properties ${ }^{17}$ and the position of the associated dip. Hence, our structure's design is not carried out by simple numerical 
minimization of the average reflectance but is rather obtained through judicious design of the interplay of the two mechanisms using our theoretical model.

\section{Experiment}

To demonstrate the doubly-resonant metasurface AR-coating experimentally, we fabricate an optimized metafilm based on a Si nanobeam array and measure its reflection spectrum. Using the dispersive index of silicon ${ }^{34}$ and aiming to place both reflection dips within the visible spectrum, the designed geometry for polarized light is a nanowire array with $w=80 \mathrm{~nm}, h=120 \mathrm{~nm}$, and $p$ $=180 \mathrm{~nm}$ (see Supplementary Info). The structures were fabricated by electron-beam lithography. Si substrates were spin-coated with an 80-nm-thick layer of hydrogen silsesquioxane negative ebeam resist (HSQ, 4\% in methyl isobutyl ketone), and baked for 45 min at $90{ }^{\circ} \mathrm{C}$. Lines were fabricated in the HSQ by exposure using a JEOL JBX 6300 lithography system (100 kV, dose 1500-3500 $\mu \mathrm{C} / \mathrm{cm}^{2}$ ) and development in tetramethylammonium hydroxide (TMAH 25\%, $120 \mathrm{~s}$ ). The pattern was then transferred into the Si by a two-step reactive-ion etching procedure using $\mathrm{C}_{2} \mathrm{~F}_{6}$ and $\mathrm{Cl}_{2} / \mathrm{HBr}$, respectively. Finally, the cross-linked HSQ mask was removed using $\mathrm{HF}$ (5\% in $\mathrm{H}_{2} \mathrm{O}$ ). The resulting nanobeam array is composed of smooth $\mathrm{Si}$ beams that are uniform in size across a large area (Fig. 3a). The focused-ion-beam (FIB) cross-section shows slight slanting of the walls with a width that is changing from top to bottom from about 65 to $115 \mathrm{~nm}$ (Fig. 3b). The height of the beams is about $145 \mathrm{~nm}$, as determined by atomic-force microscopy (AFM) (Fig. 3c). We measure the local reflectance spectrum with a Nikon C1Si Confocal Microscope equipped with a Princeton Instruments SpectraPro 2300i spectrometer and 
(a)

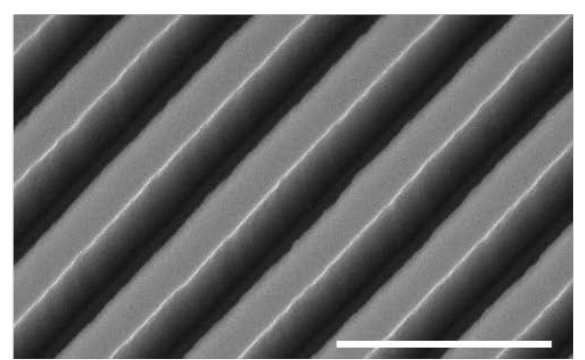

(b)

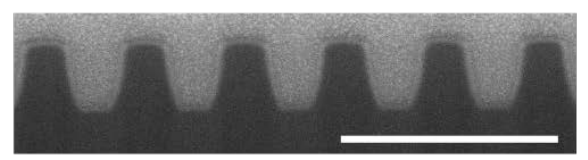

(c)

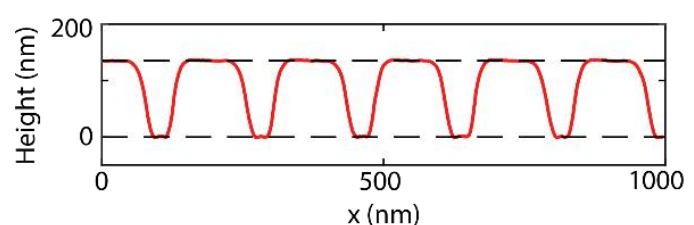

(d)

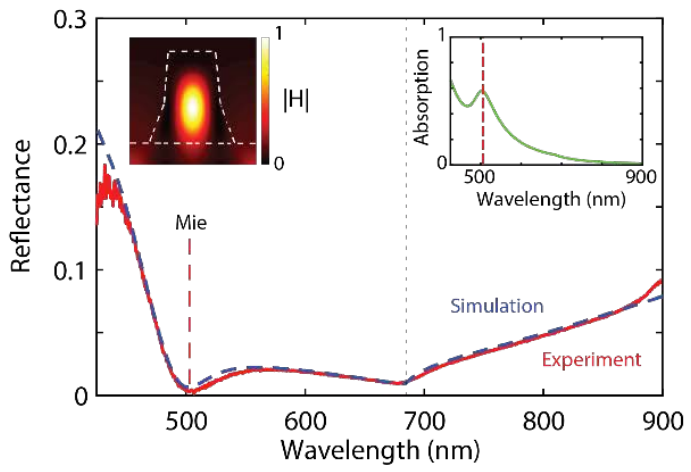

Figure 3. Fabrication and optical properties of a Mie-resonant antireflection coating composed of Si nanobeams. (a) Tilted SEM image of the Si nanobeam array. (b) SEM image of a FIB cross section of the same nanobeam array shows slight slanting of the side walls. The scale bar in (a) and (b) is $400 \mathrm{~nm}$. (c) Depth profile analysis obtained from an AFM line-scan. (d) Experimental (red) and simulated (dashed blue) reflection spectra. Insets: magnetic field amplitude profile and absorption within the nanobeam volume. The dashed vertical red line denotes the location of the Mie resonance while the dashed black line indicates the onset of diffraction in the substrate.

PIXIS CCD-detector. We use a 20X objective (Nikon, NA = 0.4) and a $100 \mu \mathrm{m}$ pinhole to analyze the reflectivity of the nanobeam array across the 425-900 nm spectral range. We employ a silvercoated mirror (Thorlabs PF10-03-P01) as a reference. Also, the microscope's aperture diaphragm 
(A-stop) is closed to ensure the smallest incident angle possible, and a polarizer in the collection path is used to analyze just the TE polarized light reflection.

The measured reflectance spectrum (red, Fig. 3d) experimentally demonstrates the doubledip in reflectance as a result of the doubly-resonant behavior of the metasurface AR coating. First, a minimum located at $500 \mathrm{~nm}$ due to the magnetic dipole resonance in the individual wires is present. To corroborate this, the simulated absorption within the nanowires is shown as an inset, which exhibits a clear peak around $\lambda=500 \mathrm{~nm}$ due to resonant absorption. The magnetic field amplitude at resonance is also shown, with the characteristic magnetic dipolar profile clearly observable. Second, a spectrally-broad minimum is observed between $600<\lambda<800 \mathrm{~nm}$ due to the FP resonance. The kink in reflectance close to $700 \mathrm{~nm}$ is due to a Rayleigh anomaly at the diffraction edge. Using the actual dimensions and shape extracted from FIB cross-sections and AFM line scans to account for fabrication imperfections, it is possible to compare the experimental spectrum to its FDTD-simulated counterpart using the dispersive index of silicon $^{34}$. The resulting spectrum (blue dashed, Fig. 3d) shows very good correspondence to the experimental spectrum. Broadband AR is obtained from two separate spectrally engineered resonant modes within the same metasurface coating, with an averaged experimental reflectance as low as $3.9 \%$ over the wavelength range from 425 - $900 \mathrm{~nm}$ (weighted for the AM 1.5 solar spectrum). The experimentally observed double-dip is not as evident as in the theory (Fig. 2d) and this can be understood by assessing the impact of fabrication imperfections on the reflectance of our structure, as discussed in depth within the next section.

The results thus far demonstrate how anisotropic structures - like the arrays of Si nanowires in Fig. 3a - can combine two resonant mechanisms to obtain broadband AR for polarized light. Most practical applications, however, require efficient AR for unpolarized light. To demonstrate the 
(a)

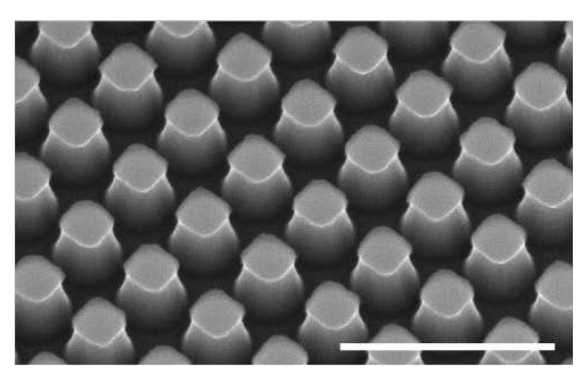

(b)

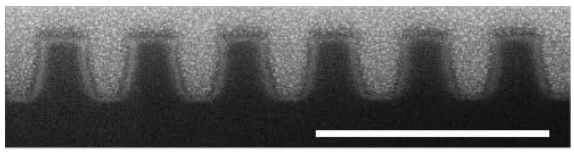

(c)

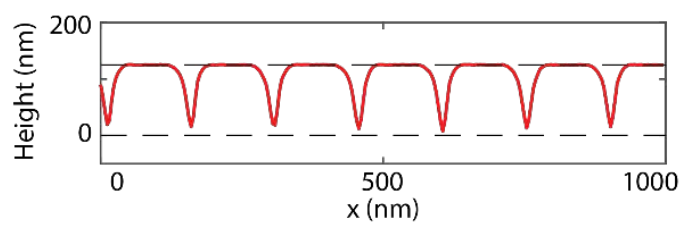

(d)

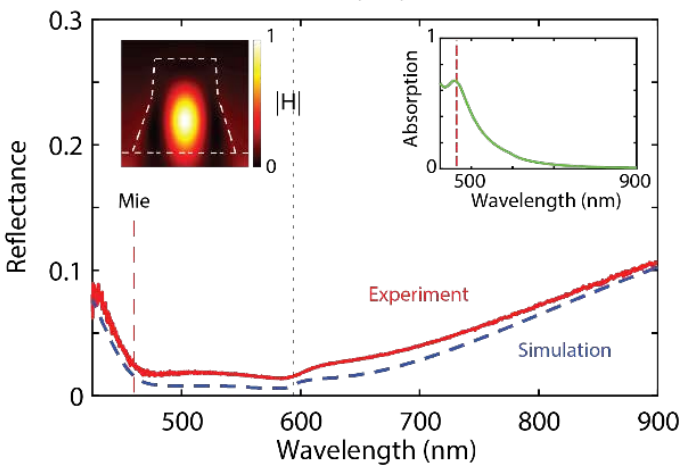

Figure 4. Fabrication and optical properties of a Mie-resonant antireflection coating composed of Si nanopillars. (a) Tilted SEM image of the nanopillar metasurface. (b) SEM image of a FIB cross section of the nanopillar array. The scale bar in (a) and (b) is $400 \mathrm{~nm}$. (c) Depth profile analysis obtained from AFM line-scan. (d) Experimental reflectance spectrum for unpolarized light (red) and simulated (dashed blue) counterpart using the dispersive index of $\mathrm{Si}^{34}$. Insets: magnetic field amplitude profile and absorption within the nanopillar volume. The dashed vertical red line denotes the spectral location of the Mie resonance while the dashed black line indicates the onset of diffraction in the substrate.

multi-resonant metasurface concept for unpolarized light, we design an array of square-based nanopillars with $w=90 \mathrm{~nm}, h=115 \mathrm{~nm}$, and $p=150 \mathrm{~nm}$. Figure 4 displays the structural and optical characterization of the fabricated nanopillar array. Similar to the nanowires array, SEM 
and FIB analysis reveal a good sample uniformity with some slanting of the sidewalls (Figs. 4ab). Combining FIB cross sections and AFM line scans (Fig. 4c) the measured as-fabricated dimensions are $h=125 \mathrm{~nm}$ and a width varying from $75 \mathrm{~nm}$ at the top of the pillars to $115 \mathrm{~nm}$ at the bottom. As with the nanobeam array, the slanting strongly affects the optical response of the metafilm (Fig. 4d). Although the double-dip is not visible, it is still possible to distinguish the same features that characterize the nanobeam array reflectance spectrum: around $450 \mathrm{~nm}$ the Mie mode (shown as inset) is producing an extra dip in the broad spectral reflectance curve due to the FP resonance. A kink around $600 \mathrm{~nm}$ again marks the onset of diffraction into the substrate. The absence of the double-dip is due to the fact that the two pathways are spectrally closer compared to the case of the nanobeam array. However, this proves to be beneficial to the overall performance of our structure showing a 4.1\% AM1.5-averaged reflectance for unpolarized light. Again, the agreement between simulation and experiment is good and only small deviations result from a non-perfect capture of the exact shape/dimensions of the metafilm. Altogether, the good agreement seen between experiment and simulations suggests the value of multi-resonant metasurfaces in suppressing reflection both for polarized and unpolarized light.

\section{Discussion}

The overall good agreement between experiment and simulations could only be obtained by taking into account the slanting of the sidewall of the Mie resonators produced by the reactive-ion etching process. Its notable impact on the reflectance properties raises the question whether the sidewall slant can be engineered to further increase the performance of Mie-resonant AR coatings. Figure 5a shows the simulated spectral reflectance (color) of an as-designed Si nanobeam array (w $=80$ $\mathrm{nm}, \mathrm{h}=120 \mathrm{~nm}$, and period $\mathrm{p}=180 \mathrm{~nm}$ ) as a function of the sidewall 
(a)

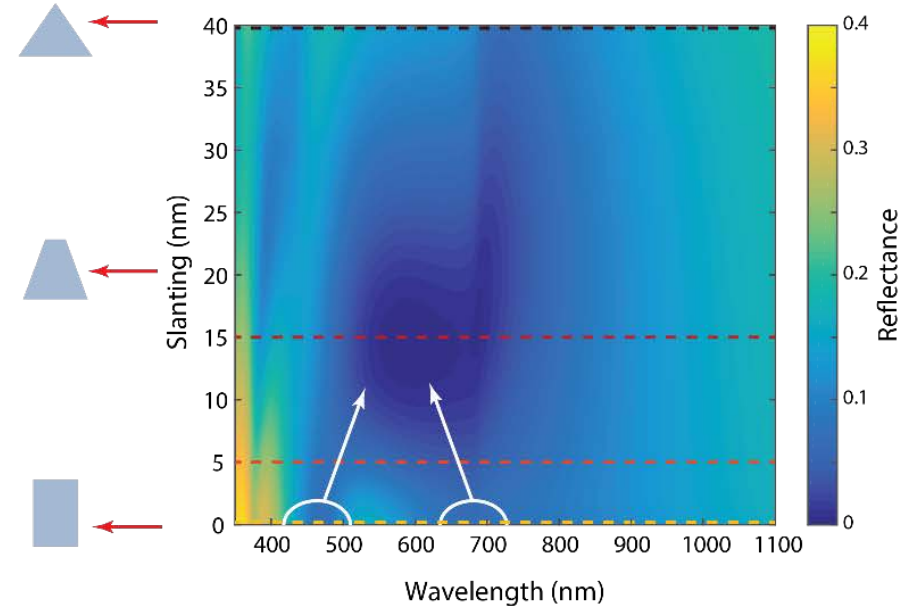

(b)

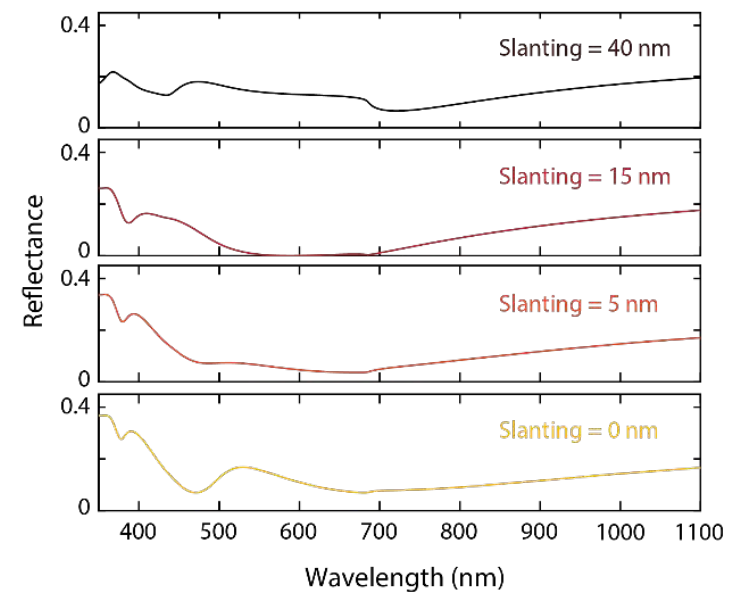

Figure 5. Impact of the sidewall slant-angle of Mie resonators on the spectral reflectance properties. (a) Dependence of the spectral reflectance (color) for an array of Si nanobeams ( $w=$ $80 \mathrm{~nm}, h=120 \mathrm{~nm}$, period $180 \mathrm{~nm}$ ) on the slant-angle of the sidewalls. (b) Line cuts through (a) for straight walls (yellow, $0 \mathrm{~nm}$ slanting), $5 \mathrm{~nm}$ slanting (orange), $15 \mathrm{~nm}$ slanting (red) and $40 \mathrm{~nm}$ slanting (black).

slant angle. Specifically, the sidewalls of the structures were slanted while keeping both the height and the width at half height constant. Figure 5b shows cross cuts of resulting trapezoidal crosssectional shapes (a) for straight walls (slanting of $0 \mathrm{~nm}$ ) and for increasing values of slanting. The striking feature in Fig. 5a is that the two dips, result of the different resonant mechanisms, merge into a broad single dip with increasing slanting. In fact, the reflection at the merged minimum (at $15 \mathrm{~nm}$ slanting, corresponding to a slanting angle of 14 degrees) is remarkably low. To investigate this phenomenon, the effects of slanted sidewalls on the resonant and on the direct pathways are analyzed separately. The Mie-resonant pathway is broadening and red-shifting with increased slanting (Supplementary Information). The characteristic displacement current loops of the magnetic dipole mode move into the substrate as the sidewalls acquire a slant. As a result, the resonator-to-substrate coupling is stronger, and the leakage rate into the substrate increases, giving rise to a broadening of the resonance. In addition, the lateral dimension of the currents loops 
increases with slanted sidewalls. As a consequence, the effective resonator width increases and its resonance wavelength red-shifts. On the other hand, the FP resonant pathway through the slanted nanobeam array can be approximated by treating the wire cross-section as $N$ effective layers with increasing effective indices. Changing the slant angle of the sidewalls is accounted for by grading the set of effective indices with the height above the surface (see Supplementary Information). The consequences of slanting are a blue-shift of the first FP reflectance minimum and a general reduction in reflection as a result of the graded-index. The red and white dashed lines of Fig. 1c move closer together with increased slanting of the side walls. Note that structures with slightly slanted sidewalls perform better than both perfect structures with straight walls and completely slanted ones (see Fig. 5b), which explains the low broadband reflectance that was observed experimentally. Thus, while the fabrication imperfections make the observation of the two reflection minima more challenging, the resulting broadband lower reflectance is highly beneficial for practical applications.

\section{Conclusion}

In conclusion, we provide a framework to model, design, and fabricate semiconductor metafilms that combine Mie and Fabry-Pérot resonances to attain a broadband anti-reflection response. Specifically, we presented the working principle of our metasurfaces in a rather intuitive way supporting our explanation with CMT and showing a strong agreement between theory and FDTD simulations. Using the insights gained from CMT it is possible to optimize the interplay of the two interfering pathways by changing the structures' geometry and hence engineer a double-dip in reflection within the visible spectrum. It is important to stress that this design can be tailored not only for different materials but also for other frequency ranges, with several potential applications 
(solar cells, image sensors, detectors, displays components etc.). Both nanobeam- and nanopillararrays are fabricated and show an experimental AM1.5-averaged reflectance as low as 3.9\% and 4.1\% for polarized and unpolarized light, respectively. Detailed structural analysis of the fabrication imperfections and their impact on optical properties revealed that slightly slanted structures give rise to a broadening and spectral overlap of the Mie and Fabry-Pérot resonances. As a result, we can achieve even lower and more broadband anti-reflection performance than for structures with perfectly straight sidewalls. Finally, we note that the conceptual demonstration of multi-resonant metasurface AR coatings in this work could be up-scaled to practical applications with fabrication techniques such as substrate conformal imprinting lithography ${ }^{35}$, nanoimprint lithography ${ }^{36}$ or rolling photolithography ${ }^{37}$, which enable large-area printing of engineered nanometer-scale structures in an inexpensive manner.

\section{ASSOCIATED CONTENT}

The following files are available free of charge.

Supplementary Information: Anti-reflection high-index metasurfaces combining Mie and FabryPérot resonances (PDF)

\section{AUTHOR INFORMATION}

\section{Corresponding Author}

*E-mail: *brongersma@stanford.edu

\section{Present Addresses}

†Andrea Cordaro: Center for Nanophotonics, AMOLF, Science Park 104, 1098 XG Amsterdam, The Netherlands 


\section{Author Contributions}

The manuscript was written through contributions of all authors. All authors have given approval to the final version of the manuscript.

\section{ACKNOWLEDGMENT}

The research was funded by a gift from ENEL Green Power. Part of this work was performed at the Stanford Nano Shared Facilities (SNSF) and Stanford Nanofabrication Facility (SNF), supported by the National Science Foundation under award ECCS-1542152. S.R. was supported by a research grant (VKR023371) from VILLUM FONDEN. J.vdG. was supported by a Rubicon postdoctoral fellowship by the Netherlands Organization for Scientific Research (NWO).

\section{REFERENCES}

1) J. W. S. Rayleigh, "On reflection of vibrations at the confines of two media between which the transition is gradual,” P. Lond. Math. Soc. s1-11, 51-56 (1879).

2) F. W. Sexton, "Plasma nitride AR coatings for silicon solar cells," Sol. Energy Mater. 7, 114 (1982).

3) J. Zhao and M. A. Green, "Optimized antireflection coatings for high-efficiency silicon solar cells," IEEE Transactions on Electron Devices 38, 1925-1934 (1991).

4) J. Zhao, A. Wang, P. Altermatt, and M. A. Green, "Twenty-four percent efficient silicon solar cells with double layer antireflection coatings and reduced resistance loss," Appl. Phys. Lett. 66, 3636-3638 (1995).

5) S. Chhajed, M. F. Schubert, J. K. Kim, and E. F. Schubert, "Nanostructured multilayer graded-index antireflection coating for Si solar cells with broadband and omnidirectional characteristics,” Appl. Phys. Lett. 93, 251108 (2008)

6) X. Yan, D. J. Poxson, J. Cho, R. E. Welser, A. K. Sood, J. K. Kim, and E. F. Schubert, "Enhanced omnidirectional photovoltaic performance of solar cells using multiple-discretelayer tailored- and low-refractive index anti-reflection coatings,” Adv. Funct. Mater. 23, 583-590 (2013).

7) J. Zhu, Z. Yu, G. F. Burkhard, C.-M. Hsu, S. T. Connor, Y. Xu, Q. Wang, M. McGehee, S. Fan, and Y. Cui, "Optical absorption enhancement in amorphous silicon nanowire and nanocone arrays,” Nano Lett. 9, 279-282 (2009). 
8) M. L. Brongersma, Y. Cui, and S. Fan, "Light management for photovoltaics using highindex nanostructures,” Nat. Mater. 13, 451-460 (2014).

9) S. Jahani and Z. Jacob, “All-dielectric metamaterials,” Nat. Nanotechnology 11, 23-36 (2016).

10) J. van de Groep, P. Spinelli, and A. Polman, "Single-step soft-imprinted large-area nanopatterned antireflection coating,” Nano Lett. 15, 4223-4228 (2015).

11) L. Cao, P. Fan, A. P. Vasudev, J. S. White, Z. Yu, W. Cai, J. A. Schuller, S. Fan, and M. L. Brongersma, "Semiconductor nanowire optical antenna solar absorbers," Nano Lett. 10, 439445 (2010).

12) L. Cao, P. Fan, E. S. Barnard, A. M. Brown, and M. L. Brongersma, “Tuning the color of silicon nanostructures,” Nano Lett. 10, 2649-2654 (2010).

13) A. B. Evlyukhin, C. Reinhardt, A. Seidel, B. S. Luk’yanchuk, and B. N. Chichkov, “Optical response features of Si-nanoparticle arrays,” Phys. Rev. B 82, 045404 (2010).

14) A. I. Kuznetsov, A. E. Miroshnichenko, M. L. Brongersma, Y. S. Kivshar, and B. Luk’yanchuk, “Optically resonant dielectric nanostructures,” Science 354 (2016).

15) C. Bohren and D. Huffman, Absorption and Scattering of Light by Small Particles, Wiley Science Series (Wiley, 2008).

16) T. J. Kippenberg, A. L. Tchebotareva, J. Kalkman, A. Polman, and K. J. Vahala, "Purcellfactor-enhanced scattering from Si nanocrystals in an optical microcavity,” Phys. Rev. Lett. 103 (2009).

17) J. van de Groep and A. Polman, "Designing dielectric resonators on substrates: Combining magnetic and electric resonances,” Opt. Express 21, 26285-26302 (2013).

18) P. Spinelli, M.Verschuuren, and A. Polman, "Broadband omnidirectional antireflection coating based on subwavelength surface Mie resonators,” Nat. communications 3, 692 (2012).

19) Z. Wang, R. Zhang, S. Wang, M. Lu, X. Chen, Y. Zheng, L. Chen, Z. Ye, C. Wang, and K. Ho, "Broadband optical absorption by tunable Mie resonances in silicon nanocone arrays," Sci. reports 5, 7810 (2015).

20) Z. Yu, A. Raman, and S. Fan, "Fundamental limit of nanophotonic light trapping in solar cells,” Proc. Natl. Acad. Sci. 107, 17491-17496 (2010).

21) K. X. Wang, Z. Yu, S. Sandhu, and S. Fan, "Fundamental bounds on decay rates in asymmetric single-mode optical resonators,” Opt. Lett. 38, 100-102 (2013).

22) K. X. Wang, Z. Yu, S. Sandhu, V. Liu, and S. Fan, "Condition for perfect antireflection by optical resonance at material interface,” Optica 1, 388-395 (2014).

23) H. Haus, Waves and fields in optoelectronics, Prentice-Hall Series in Solid State Physical Electronics (Prentice Hall, Incorporated, 1984).

24) S. Fan,W. Suh, and J. D. Joannopoulos, “Temporal coupled-mode theory for the Fano resonance in optical resonators,” J. Opt. Soc. Am. A 20, 569-572 (2003).

25) Z. Ruan and S. Fan, "Temporal coupled-mode theory for Fano resonance in light scattering by a single obstacle,” The J. Phys. Chem. C 114, 7324-7329 (2010). 
26) T. K. Gaylord, W. E. Baird, and M. G. Moharam, "Zero-reflectivity high spatial-frequency rectangular-groove dielectric surface-relief gratings,” Appl. Opt. 25, 4562-4567 (1986).

27) D. L. Brundrett, E. N. Glytsis, and T. K. Gaylord, "Homogeneous layer models for highspatial-frequency dielectric surface-relief gratings: conical diffraction and antireflection designs," Appl. Opt. 33, 2695-2706 (1994).

28) M. E. Motamedi, W. H. Southwell, and W. J. Gunning, "Antireflection surfaces in silicon using binary optics technology,” Appl. Opt. 31, 4371-4376 (1992).

29) P. E. Landreman, H. Chalabi, J. Park, and M. L. Brongersma, "Fabry-Perot description for Mie resonances of rectangular dielectric nanowire optical resonators,” Opt. Express 24, 29760-29772 (2016).

30) Lumerical Inc. http://www.lumerical.com/tcad-products/fdtd/

31) U. Fano, "Sullo spettro di assorbimento dei gas nobili presso il limite dello spettro d'arco," Il Nuovo Cimento (1924-1942) 12, 154-161 (1935).

32) A. E. Miroshnichenko, S. Flach, and Y. S. Kivshar, "Fano resonances in nanoscale structures,” Rev. Mod. Phys. 82, 2257-2298 (2010).

33) B. Luk'yanchuk, N. I. Zheludev, S. A. Maier, N. J. Halas, P. Nordlander, H. Giessen, and C. T. Chong, "The Fano resonance in plasmonic nanostructures and metamaterials," Nat. Materials 9, 707-715 (2010).

34) E. Palik, Handbook of Optical Constants of Solids (Academic Press, 1998).

35) M. A. Verschuuren, M. Megens, Y. Ni, H. van Sprang, and A. Polman, "Large area nanoimprint by substrate conformal imprint lithography (SCIL),” Adv. Opt. Technol. 6, 243264 (2017).

36) S. Y. Chou, P. R. Krauss, and P. J. Renstrom, "Nanoimprint lithography,” J. Vac. Sci. \& Technol. B: Microelectron. Nanometer Struct. Process. Meas. Phenom. 14, 4129-4133 (1996).

37) B. Kobrin, E. S. Barnard, M. L. Brongersma, M. K. Kwak, and L.J. Guo, "Rolling mask nanolithography: the pathway to large area and low-cost nanofabrication," Proc. SPIE Vol. 8249, 824900 (2012). 